\title{
A Novel Immuno Chromatography Based Rapid Intraoperative PTH Detection Device Reduce the Time for Identifying Parathyroid Tissue from Thyroid Nodules
}

\author{
Xiaoning Wang*1, Yafei Liư ${ }^{1}$, Yizeng Wang ${ }^{1}$, Dongyang $\mathrm{Li}^{1}$, Xianghui $\mathrm{He}^{1}$ and Lijun Song ${ }^{2}$ \\ ${ }^{1}$ Department of General Surgery, Tianjin Medical University, China \\ ${ }^{2}$ Department of General Surgery, Weihaiwei People's Hospital, China
}

Received: March 18, 2018; Published: April 05, 2018

*Corresponding author: Xianghui He, Department of General Surgery, Tianjin Medical University General Hospital, Tianjin Medical University, 154 Anshan Road, Tianjin 300052, China, Tel: 8613920778663; Email: humphreyhe@163.com

Abstract

Intra Operative PTH detection is a feasible method to distinguish parathyroid tissue from non-parathyroid tissue during parathyroidectomy. Following the wide application of intra Operative PTH detection, many efforts were put to reduce the turnaround time. We report a case of parathyroid adenoma combined with thyroid nodules on a 63-year-old patient, and successfully identify different tissue types during surgery by a novel immune Chromatography based rapid intra Operative PTH detection device.

Keywords: Parathyroidectomy; Parathyroid Tissue; Intra Operative PTH Detection

\section{Introduction}

During parathyroidectomy, it is at times difficult for surgeons to visually differentiate parathyroid tissue from non-parathyroid tissue, including thyroid nodule, lymph node, and fat tissue. Although intra-operative frozen section analysis is the gold standard to distinguish different tissue type, more surgeons nowadays choose to aspirate the suspected parathyroid tissue and detect the PTH value in the puncture fluent to solve the problem [1-3]. This approach has a turnaround time about 15-30 minutes, much frequent than frozen section analysis [4]. Much efforts were put to modify the procedure and the detection device to reduce the turnaround time. Here, we report to apply a novel immune Chromatography based rapid intra Operative PTH detection device in a case of parathyroid adenoma combined with thyroid nodules, with a much shorter turnaround

\section{Clinical Case}

A 63-year-old female patient was diagnosed as primary hyperparathyroidism according to her complaints and laboratory evaluation. However, the preoperative high-resolution ultrasound examination indicated hypo echoic nodules posterior to lower pole of both left and right thyroid lobes, suggesting the nodules originated from parathyroid gland. The patient was operated and during intra Operative exploration, a neoplasm was observed behind the left-lower pole of thyroid gland inside true capsule, we supposed that it might be a rare case of parathyroid adenoma inside of thyroid gland, but still could not exclude the possibility of thyroid nodules. In order to distinguish the tissue type, we planned to apply a novel developed immune Chromatography based rapid intra operative PTH detection device (Biota Diagnostics, Wuhan, after aspiration of suspected tissue, the diluted puncture fluent were dropped on the sample well of the test card.

The device could report the PTH concentration within 8 minutes. The result suggested that the suspected neoplasm was not originated from parathyroid for a PTH level lower than 10pg/ $\mathrm{ml}$. A rapid PTH detection was also performed for the neoplasm observed posterior right-lower pole of thyroid gland, and the result also suggested a non-parathyroid tissue. The bilateral parathyroid exploration was performed, and another suspected parathyroid tissue was observed posterior left-upper-pole of thyroid gland, The PTH level was over $3000 \mathrm{pg} / \mathrm{ml}$ for the puncture fluent, suggesting as parathyroid adenoma tissue. The dissected tissue was sending for frozen pathology examination, and the result confirmed that the first two neoplasms explored were thyroid nodules and the third was parathyroid adenoma. Therefore, our application of rapid PTH detection system achieved a fast identification of parathyroid adenoma during surgery, confirmed a full removal of parathyroid adenoma, and avoids unnecessary exploration and excision of healthy tissue. 


\section{Discussion}

In current clinical practice, the PTH detection is done by clinical lab. Due to the distance between operation room and clinical lab, the detection time cost by laboratory staff, and other unpredictable factors, it is hard to receive the results within 10 minutes theoretically. Here, we used the novel PTH detection device in the operation room and received results within 8 minutes for whole process, much shorter than other detection method. The application of this technique can shorten the operation time, reduce the operative complications, decline the cost of operation, and improve the utilization ratio of operation room [5]. Recently, several rapid PTH detection methods are introduced to overcome the time-consuming disadvantage of conventional PTH detection system. Benjamin C et al. [6] developed a modified PTH assay protocol that achieved identification of parathyroid tissue within 60 seconds of measurement onset.

However, this technique requires a skilled and trained laboratory technician to present in the operation room, adding extra cost for training and limiting widespread of this protocol in other institutions. Veronique Jarring et al. introduced Philips handheld magnetic system based PTH testing system with a turnaround time less than 10 minutes, but the production of reagent system is highly manual and may cause additional deviation in test results [7]. The detection device we used is similar in size to a handheld POS, and could be placed in the operation room. The theatre nurses could run the test without professional guidance after a simple training. Moreover, the production of detection device and test cards is highly industrialized, reducing the error caused by manual production. However, the rapid PTH detection system and test protocol still exist several limitations. First, the dilution process is done with $1 \mathrm{ml}$ syringe and the dilution multiple of puncture fluent may varies by different handlers; we suppose to use more accurate pipettes for dilution process to overcome this problem. An abnormal high level of antibody in the sample, such as heterophil antibody, may influence the displayed result. We will continue to improve the protocol, and promote this technique for widespread use in other situation and institutions.

\section{References}

1. Barczynski M, Golkowski F, Nawrot I (2015) The current status of intraoperative iPTH assay in surgery for primary hyperparathyroidism. Gland Surg 4(1): 36-43.

2. Cal PG, Pisano G, Loi G, Medas F, Barca L, et al. (2013) Intraoperative parathyroid hormone assay during focused parathyroidectomy: the importance of 20 minutes measurement. BMC Surg 13: 36.

3. Shawky MS (2016) Quick parathyroid hormone assays: a comprehensive review of their utility in clinical practice. Hormones (Athens) 15(3): 355-367.

4. Perrier ND, Ituarte P, Kikuchi S, Siperstein AE, Duh QY, et al. (2000) Intraoperative Parathyroid Aspiration and Parathyroid Hormone Assay as an Alternative to Frozen Section for Tissue Identification. World Journal of Surgery 24(11): 1319-1322.

5. Thielmann A, Kerr P (2017) Validation of selective use of intraoperative PTH monitoring in parathyroidectomy. Journal of Otolaryngology Head Neck Surgery 46(1): 10.

6. James BC, Nagar S, Tracy M, Kaplan EL, Angelos P, et al. (2014) A novel, ultrarapid parathyroid hormone assay to distinguish parathyroid from nonparathyroid tissue. Surgery 156(6): 1638-1643.

7. Jarrige V, Nieuwenhuis JH, Van Son JP, Martens MF, Vissers JL (2011) A fast intraoperative PTH point-of-care assay on the Philips handheld magnotech system. Langenbecks Archives of Surgery 396(3): 337-343.

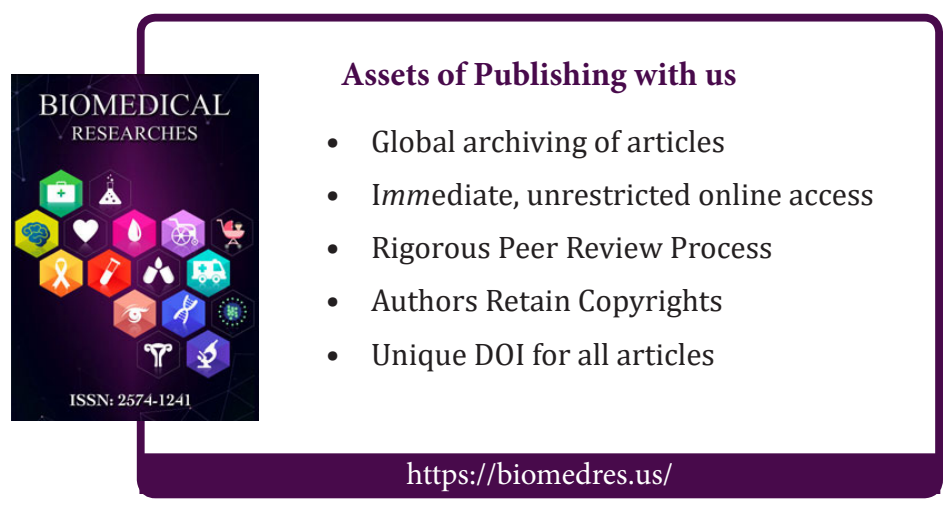

